\title{
Sur un aspect méconnu du problème de l'eau (1)
}

\author{
par \\ M. KEILLING
}

Si j'ai choisi un tel titre pour cette communication, c'est en raison de l'oubli qui me paraît être involontaire mais qui n'en est pas moins systématique d'une notion fondamentale: c'est le rôle de l'eau de boisson des animaux en matière de production.

Si l'on prend à distance, le temps de réfléchir sur la situation de notre élevage, on ne peut manquer d'être frappé de l'immense mécontentement que manifestent à chaque instant les milieux intéressés. Les planificateurs contemporains, les chercheurs qui se penchent sur l'économie et les techniques de production animale ont certes encouragé et perfectionné nombre de facteurs qui dominent la bonne évolution des élevages et cependant n'auraient-ils pas oublié ou négligé ce facteur essentiel: l'eau que boivent les animaux ? Ce qui reviendrait à considérer, tous autres progrès acquis et vulgarisés, que la qualité de l'eau deviendrait le facteur dominant, la cause ou l'une des causes des difficultés présentes.

Certes, dans d'autres voies, sélection, alimentation, insémination artificielle, des progrès considérables furent obtenus au cours des dernières années, mais qu'en est-il pour l'eau?

Si vous voulez bien prendre connaissance de l'important volume intitulé "Rapport général de la Commission de l'eau du $\mathrm{V}^{\mathrm{e}}$ Plan » vous constaterez que la quantité d'eau de boisson des animaux ne figure pas à l'inventaire des besoins. Tout se passe comme s'il n'était pas question, pour nos animaux domestiques, d'absorber une eau potable. Et pourtant, ils absorbent environ 1 million de mètres cubes par jour, que le liquide soit potable ou non!

Cependant, dans les traités d'élevage, il n'est question que d'eau potable! Mais il convient de se demander où se trouvent les troupeaux par rapport à l'eau potable?

(1) C.R. Acad. Agr. $-1968-\mathrm{n}^{\circ} 5-368$. 
Tout d'abord, examinons la carte de la répartition des adductions d'eau potable en comparaison avec la carte de la densité de population d'animaux domestiques: une constatation évidente apparaît, c'est dans les régions les moins riches en animaux que se trouve la plus grande densité d'adduction d'eau potable.

Ailleurs, en dehors des réseaux d'adduction, il est bien évident qu'il ne peut s'agir que d'eau de puits ou de mares superficielles, plus ou moins polluées, et il est aussi probable que dans les régions équipées de réseaux d'eau potable, la boisson des animaux ne provient pas obligatoirement de ceux -ci ?

Dans le rapport de la commission de l'eau est mentionnée la toxicité pour les enfants en bas âge des eaux riches en sels de l'acide nitrique. Cette indication bien connue des pédiatres semble l'être beaucoup moins des éleveurs, encore que, dans l'époque contemporaine, l'allaitement artificiel des veaux par exemple, fasse intervenir, dès le premier âge, l'eau de l'exploitation dans l'alimentation des jeunes animaux.

La littérature scientifique des Etats-Unis d'Amérique, commence à fournir à cet égard une documentation importante et dont la lecture donne à réfléchir.

C'est ainsi, par exemple, que dans l'Iowa, en 1945, l'étude des enfants cyanosés fait apparaître l'utilité du dosage des nitrates dans l'eau de puits des fermes de la région : 20 p. 100 des puits examinés sont considérés comme dangereux pour les enfants en bas âge... La divagation des résidus humains et animaux conduit à la contamination des puits par les sels ammoniacaux et les nitrates. Ceux-ci deviennent, par réduction dans l'intestin grêle, des nitrites qui agissent sur l'hémoglobine du sang ou font de la méthémoglobine provoquant une intoxication plus ou moins importante qui se répercute, outre l'aspect cyanosé, sur la consistance musculaire et sur la croissance des jeunes enfants. C'est ainsi que l'on constate la disparition des protozoaires du rumen chez les vaches ayant absorbé 9 grammes de nitrate pour 100 kilogrammes dans leur alimentation et ces considérations m'ont conduit à rechercher, dans de nombreuses localités, quelle était la teneur en nitrates des points d'eau où s'abreuvaient les animaux.

J'ai obtenu, par exemple, les résultats suivants, en janvier 1968, dans une région laitière de plaine, à l'Est de Paris :

\begin{tabular}{|c|c|c|c|}
\hline & & & $\mathrm{NO}_{3} \mathrm{~K}$ \\
\hline & & & $\mathrm{mmg} / \mathrm{l}$ \\
\hline Exploitation $\mathrm{n}^{\circ}$ & 1 & Puits individuel & 110 \\
\hline & 3 & $\gg \quad 》$ & 120 \\
\hline & 4 & $»$ & 740 \\
\hline & 7 & $»$ & + de $1 \mathrm{~g}$ \\
\hline & 8 & $»$ & 190 \\
\hline
\end{tabular}




\begin{tabular}{|c|c|c|c|c|}
\hline & 9 & $»$ & $»$ & 125 \\
\hline & 10 & $»$ & $»$ & 160 \\
\hline & 11 & $»$ & $»$ & 200 \\
\hline & 14 & $»$ & $»$ & 400 \\
\hline & 15 & $»$ & $»$ & 130 \\
\hline & 16 & » & $»$ & 92 \\
\hline & 18 & $》$ & $»$ & 280 \\
\hline & 19 & $»$ & $»$ & 250 \\
\hline & 21 & $»$ & $"$ & 25 \\
\hline & 22 & ", & $»$ & 270 \\
\hline & & & & $\mathrm{No}_{3} \mathrm{~K}$ \\
\hline & & & & $\mathrm{mmg} / \mathrm{l}$ \\
\hline Exploitation $n^{\circ}$ & 2 & Eau de & concession & 2 \\
\hline & 5 & $»$ & $»$ & 14 \\
\hline & 6 & $»$ & $»$ & 2,5 \\
\hline & 17 & $»$ & $»$ & traces \\
\hline & 20 & $»$ & $»$ & 0 \\
\hline & 23 & $»$ & $»$ & 22 \\
\hline & 24 & $»$ & $»$ & 22 \\
\hline & 13 & Eau de & pluie récupérée & 5 \\
\hline & 12 & Eau de & mare & 72 \\
\hline
\end{tabular}

S'il m'est permis de faire ici un retour en arrière, d'un siècle environ, je rappellerai qu'en 1857 notre prédécesseur, J.B. Boussingault, enquêtait sur la teneur en nitrates des eaux des puits de Paris et enregistrait les données analytiques ci-après:

$\begin{array}{cc} & \text { Equivalent } \\ \text { à Nitrate de } \\ \text { Potasse } \\ \text { Lar litre } \\ \text { d'eau }\end{array}$

Rue du Pas-de-la-Mule ................ 0,6071 g

Rue Saint-Martin, $294 \ldots \ldots \ldots \ldots \ldots \ldots \ldots$ 0,2232 g

Rue des Noyers, $70 \ldots \ldots \ldots \ldots \ldots \ldots \ldots$ 1,500. $\mathrm{g}$

Rue des Vieilles Etuves .............. 0,4743 g

Faubourg Saint-Honoré, $66 \ldots \ldots \ldots \ldots \ldots \ldots$ 0,6701 g

Rue de Sèvres, $10 \ldots \ldots \ldots \ldots \ldots \ldots \ldots \ldots \ldots \ldots$ 0,4742 g

Rue Levert, 14, Belleville ............. 1,5464 g

Rue Mouffetard, $132 \ldots \ldots \ldots \ldots \ldots \ldots \ldots \ldots . \ldots \ldots$ 0,2165 g

Place Royale ...................... 0,4124 g

Cimetière Montmartre .............. 1,5258 g

et il commentait sévèrement la pratique de la Boulangerie de l'Assistance Publique, rue Scipion, qui délivrait aux malades des 
hôpitaux des pains riches en nitrate amenés par l'eau employée pour leur confection (l'équivalent de $0,31 \mathrm{~g} / \mathrm{l}$ de nitrate de potasse).

Les eaux d'abreuvement du bétail ont des teneurs très voisines de celles qu'enregistrait jadis Boussingault et les pratiques de fertilisation azotée, d'intensification de production nous conduiront, si l'on n'y prend garde, à des teneurs encore plus élevées, alors que nous augmenterons par l'alimentation moderne le rôle que joue l'eau dans la croissance des jeunes animaux. Il ne s'agit certes pas d'une toxicité aiguë, car l'alarme serait déjà donnée et le danger perçu, mais d'une toxicité faible, diffuse, variable, suivant le régime des pluies et les points d'eau dont il s'agit, mais, dans l'économie résultant des croissances et des assimilations le danger d'une telle toxicité me paraît devoir être souligné. 